\title{
Blood Neutrophil-to-Lymphocyte Ratio and Urine IL-8 Levels Predict the Type of Bacterial Urinary Tract Infection in Type 2 Diabetes Mellitus Patients
}

This article was published in the following Dove Press journal: Infection and Drug Resistance

Fatemeh Saheb Sharif-Askari (D) Narjes Saheb Sharif-Askari (iD)

Adnane Guella $\mathbb{D D}^{2}$

Ali Alabdullah (D) ${ }^{1, *}$

Hour Bashar Al Sheleh (D) ${ }^{1} *$

Afnan Maher Hoory AIRawi (iD ${ }^{1, *}$

Enad Sami Haddad (ID) ${ }^{1, *}$

Qutayba Hamid 1,3

Rabih Halwani (iD ${ }^{1,3,4}$

Rifat Hamoudi (D) ${ }^{1,3}$

'Sharjah Institute of Medical Research, College of Medicine, University of

Sharjah, Sharjah, United Arab Emirates;

${ }^{2}$ Department of Nephrology, University

Hospital Sharjah, Sharjah, United Arab

Emirates; ${ }^{3}$ Department of Clinical

Sciences, College of Medicine, University

of Sharjah, Sharjah, United Arab Emirates;

${ }^{4}$ Prince Abdullah Ben Khaled Celiac

Disease Research Chair, Department of

Pediatrics, Faculty of Medicine, King Saud

University, Riyadh, Saudi Arabia

*These authors contributed equally to this work
Background: Extended-spectrum B-lactamase (ESBL)-producing Escherichia coli (E. coli) and Klebsiella pneumoniae (K. pneumoniae) are the most common uropathogens causing UTI (urinary tract infection) in type 2 diabetes mellitus (T2DM). Circulatory inflammatory markers such as C-reactive protein (CRP) and neutrophil-to-lymphocyte ratio (NLR) are usually dysregulated during UTI. However, the differential regulation of these inflammatory signatures during E. coli and $K$. pneumoniae UTI in T2DM has not been determined.

Methods: A case-control study on 466 patients was performed to investigate the inflammatory signatures indicative of ESBL-E. coli and $K$. pneumoniae UTIs in T2DM. Serum CRP levels and blood NLR for these patients were determined and associated with $E$. coli and $K$. pneumoniae ESBL uropathogen using multivariate logistic regression analysis. Urinary interleukin 8 (IL-8) levels were also assessed and associated with these two UTI uropathogens in T2DM. The association of the two ESBL-uropathogens with the survival outcomes of T2DM patients was also analyzed using Cox-proportional hazard model.

Results: T2DM patients with ESBL-E. coli UTI had lower serum CRP levels (median, CRP $\mathrm{mg} / \mathrm{dL} 33.7$ vs 39.8, respectively; $\mathrm{P}=0.023$ ) and higher blood NLR (median, NLR 3.2 vs 2.6, respectively; $\mathrm{P}=0.010)$ compared to those with $K$. pneumoniae UTIs $(P<0.001)$. Moreover, in T2DM, the urinary IL-8 levels was higher in ESBL- $E$. coli compared to those with $K$. pneumoniae UTIs $(P<0.0001)$. After adjusting for confounders, including age, gender, serum albumin, hemoglobulin, leukocytes, and platelet counts, T2DM patients with blood NLR $\geq 3.5$ were at higher risk for ESBL-E. coli UTIs than ESBL-K. pneumoniae UTIs (odds ratio [OR], $3.61,95 \%$ confidence interval, $\mathrm{Cl}, 1.49-8.73$; $\mathrm{P}=0.004$ ). Moreover, T2DM patients with ESBLE. coli UTIs had higher all-cause mortality (hazard ratio [HR], 4.09; 95\%, 1.14-14.59) than those with $K$. pneumoniae UTIs.

Conclusion: Serum CRP levels, blood NLR, and IL-8 urinary levels differentiate ESBL-E. coli from $K$. pneumoniae UTIs in T2DM.

Keywords: extended-spectrum ß-lactamase, Klebsiella pneumoniae, Escherichia coli, urinary tract infection, C-reactive protein, survival

\section{Introduction}

Escherichia coli $(E$. coli) is the most common cause of community-acquired or nosocomial urinary tract infection (UTI). ${ }^{1}$ Extended-spectrum ß-lactamase (ESBL)producing Enterobacteriaceae such as E. coli and Klebsiella pneumoniae (K. pneumoniae $)^{2,3}$ are the most frequent isolate in UTIs and are resistant to many classes of antibiotics. ${ }^{4}$ Type 2 diabetes (T2DM) patients are at an increased risk for
Correspondence: Rifat Hamoudi; Rabih Halwani

College of Medicine, University of Sharjah, Sharjah, United Arab Emirates Email rhamoudi@sharjah.ac.ae; rhalwani@sharjah.ac.ae 
UTIs with these uropathogens, and $E$. coli being the most frequent isolate. ${ }^{5}$ Almost half of T2DM develop ESBL-positive UTIs that might result in acute kidney injury. ${ }^{6}$

C-reactive protein (CRP) has been used as an early biomarker predicting bacterial infections and is used as a marker to monitor antibiotic treatment of these infections. ${ }^{7}$ Recent reports have suggested the use of CRP blood levels to differentiate acute pyelonephritis (upper UTIs) from asymptomatic bacteriuria in pediatric UTIs. ${ }^{8}$ Blood neutrophil-to-lymphocyte ratio (NLR) has also been suggested to be used as a diagnostic marker for UTIs. ${ }^{9,10}$ Assessment of urine cytokine levels could reflect the type of immune responses induced during UTI, and may be associated with specific types of uropathogens. Elevated levels of urinary IL- 8 have been observed in the urine of children with acute pyelonephritis and renal scarring. ${ }^{11}$ However, studies that associate these inflammatory markers to the type of uropathogen in T2DM are lacking. Therefore, the objective of our study was to determine the inflammatory markers that may differentiate between ESBL-producing E. coli and K. pneumoniae uropathogens causing UTIs during T2DM. The association of these uropathogens with all-cause mortality of T2DM patients was also determined.

\section{Methods}

\section{Ethical Considerations}

Ethical approval was obtained from the University Hospital Sharjah Research Ethics Board (Reference No: UHS/CS/HERC/F001-2/17). All methods were performed in accordance with the relevant guidelines (Declaration of Helsinki and the Belmont Report). Written informed consent was obtained from all study participants.

\section{Study Patients}

The initial cohort included a total of 668 patients from both outpatients and inpatients diagnosed with ESBL-positive UTI (ESBL-UTI), from January 1st 2017 through 15th July 2019. ESBL-UTI diagnosis was based on positive microbiological test results and patient symptoms. Symptoms were typically acute in onset and included dysuria, urinary frequency and urgency, suprapubic or flank pain, hematuria, and/or documented fever. Inclusion criteria included adults both males and females with ESBL-positive UTI test who had complete microbiological test results and laboratory values including complete blood counts and serum CRP value. Exclusion criteria were younger than 18 years, pregnant women, and those with active malignancy. Out of the 668 ESBL-UTI patients, 466 patients had complete laboratory values and microbiology test results. Around half of these ESBL-UTI patients had T2DM ( $\mathrm{n}=264,57 \%)$. T2DM was determined using laboratory measurements of FBG, $\mathrm{HbAlc}$ and patient medical record information. Following the WHO criteria for both FBG and $\mathrm{HbAlc}$; FBG $<6.1 \mathrm{mmol} / 1$ was considered normal and $\geq 7.0 \mathrm{mmol} / \mathrm{l}$ indicated diabetes. However, $\mathrm{HbAlc}<6.5 \%$ was considered non-diabetic and $\geq 6.5 \%$ indicated diabetes. ${ }^{12}$

\section{Univariate and Multivariate Logistic Regression Analysis}

For the purpose of descriptive analysis, patient's demographic, clinical data, and laboratory test results were associated with ESBL-E. coli or K. pneumoniae UTIs. For two-way analysis of continuous variables, $t$-test or Mann-Whitney $U$-test was used depending on the skewness of data. Continuous variables were first analyzed without categorization, but a different cutoff value was used in multivariate analysis. Chi-square $\left(X^{2}\right)$ test was used for categorical variables analysis.

To investigate the inflammatory signatures indicative of UTIs in T2DM and non-T2DM subgroups, we determined the serum CRP and blood NLR levels for these patients in association with $E$. coli or $K$. pneumoniae uropathogen. The risk for E. coli or K. pneumoniae UTI diagnosis for these patients was assessed based on serum CRP values and blood NLR using multivariate logistic regression analysis, after adjusting for various cofounders.

The accuracy of the regression model was evaluated using positive predictive value, and negative predictive value. Sensitivity (percentage predicted positive among all truly positive) and specificity (percentage predicted negative among all truly negative) were also calculated. A sample size of 77 cases and 77 controls were assessed to provide $>80 \%$ power to detect a significant difference $(\alpha=0.05)$ across subgroups of ESBL- $E$. coli or ESBL- $K$. pneumoniae UTIs using $\mathrm{G}^{*}$ power software. ${ }^{13}$

\section{ELISA Analysis}

Urine IL-8 levels for T2DM were assessed using ELISA assay according to the manufacture's instructors (IL-8, Cat \# ab46032 Abcam, Cambridge, MA, USA). Each sample was assayed in triplicate and values were expressed as the mean of three measures per sample. 


\section{Survival Analysis}

The survival time for individual patients was defined as the date from diagnosis of ESBL-positive UTIs to the date of death from any cause. Surviving patients were censored at the end of the study on July 15, 2019 after survival probability was determined using Cox-proportional hazards model adjusted for various cofounders, and the resultant cumulative survival probability curve was plotted. All statistical tests were 2-sided and considered statistically significant at $P<.05$. The analysis was performed using SPSS Version 26 (IBM Corporation, Chicago, USA) and Graphpad Prism 7 (GraphPad Software Inc., San Diego, USA).

\section{Gene Set Enrichment Analysis}

To determine neutrophil-E. coli interaction, Gene Set Enrichment Analysis (GSEA) was carried out on the following data set from the NCBI's GEO database; GSE $126757,{ }^{14}$ as previously described on NF-B inflammatory pathway using the Kolmogorov-Smirnoff test. ${ }^{15}$ The data used were the RNA-seq generated from healthy human neutrophils and E. coli-stimulated human neutrophils. The related Figure is included in the supplemental material.

\section{Results}

\section{Demographic and Clinical Characteristics of the Patients}

A total of 668 patients with a confirmed diagnosis of UTI caused by either ESBL-E. coli, or ESBL-K. pneumoniae were screened during the study period between January 2017 and July 2019. Of these, 202 patients did not have pretreatment urine samples or complete laboratory test results and were therefore excluded from the primary analysis (Figure 1). The mean (SD) age of study participants was 68 (20). Almost half of the 466 recruited patients had T2DM $(n=264,57 \%)$. The mean (SD) of HbA1c in T2DM was 7.4 (3). One third of T2DM had chronic kidney disease (CKD) $(n=85,32 \%)$.

Patients were categorized into two groups: patients with ESBL-E. coli UTIs and those with ESBL-K. pneumoniae UTIs. Overall, the two groups were balanced relative to baseline characteristics and were similar with respect to serum albumin levels (Table 1). ESBL-E. coli uropathogens had lower level of resistance to $\beta$-lactam $/ \beta$-lactamase inhibitors (sensitivity of $65 \%$ vs $52 \%$ to amoxicillin-clavulanate and $92 \%$ vs $83 \%$ to piperacillin-tazobactam for ESBL- $E$. coli UTIs and ESBL-K. pneumoniae UTIs, respectively). They were also more resistance to ciprofloxacin (sensitivity of $27 \%$ vs $40 \%$ for ESBL- $E$. coli UTIs and ESBL- $K$. pneumoniae UTIs, respectively).

\section{Serum CRP Levels and Blood NLR Differentiate ESBL-E. coli from $K$. pneumoniae UTls in T2DM Patients}

Elevated serum CRP is a marker for inflammation, and its level increases during bacterial infection including UTI. ${ }^{7}$ However, this concept applies for all pathogens, including E. coli, which accounts for up to two-thirds of all UTI cases; and is the most frequent isolate in T2DM UTIs. ${ }^{5}$ There is no report differentiating the inflammatory profile of UTIs caused by ESBL-E. coli versus $K$. pneumoniae, especially in T2DM.

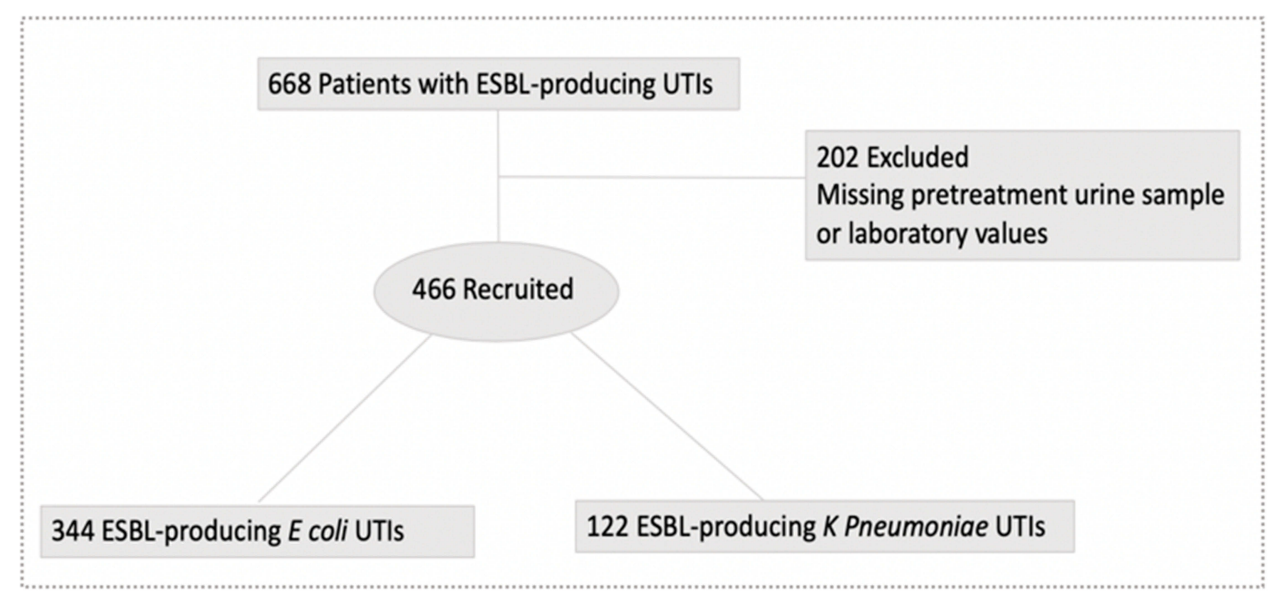

Figure I Patient recruitment and flow through study. 
Table I Demographic and Clinical Characteristics of Patients with ESBL-Positive UTIs

\begin{tabular}{|c|c|c|c|}
\hline Characteristic & $\begin{array}{l}\text { E. coli } \\
(n=344)\end{array}$ & $\begin{array}{l}\text { K. pneumoniae } \\
(n=122)\end{array}$ & $P$-value* \\
\hline $\begin{array}{l}\text { Age, mean (SD), y } \\
\text { Female } \\
\text { T2DM } \\
\text { CKD }\end{array}$ & $\begin{array}{l}67(21) \\
210(61) \\
179(63) \\
64(19)\end{array}$ & $\begin{array}{l}74(18) \\
72(59) \\
85(77) \\
53(43)\end{array}$ & $\begin{array}{l}0.001 \\
0.668 \\
0.421 \\
0.205\end{array}$ \\
\hline $\begin{array}{l}\text { Laboratory data } \\
\text { HbAIC, mean (SD), \% } \\
\text { Albumin, mean (SD), g/dL } \\
\text { Hemoglobin, mean (SD), g/dL } \\
\text { Leukocyte, mean (SD) } \\
\text { Neutrophils, mean (SD) } \\
\text { Lymphocytes, mean (SD) } \\
\text { Procalcitonin, mean (SD) } \\
\text { Platelet count, mean (SD) }\end{array}$ & $\begin{array}{l}7.25(3) \\
28.54(5) \\
10.94(1) \\
10.09(4) \\
7.30(5) \\
1.98(3) \\
0.16(0.1) \\
269(108)\end{array}$ & $\begin{array}{l}7.29(2) \\
28.28(5) \\
10.85(1) \\
9.76(4) \\
7.00(6) \\
2.82(6) \\
0.15(0.1) \\
274(14 I)\end{array}$ & $\begin{array}{l}0.959 \\
0.706 \\
0.544 \\
0.512 \\
0.741 \\
0.051 \\
0.712 \\
0.696\end{array}$ \\
\hline Antibiotic sensitivity & & & \\
\hline $\begin{array}{l}\boldsymbol{\beta} \text {-lactam/ } \boldsymbol{\beta} \text {-lactamase } \\
\text { inhibitor } \\
\text { Amoxicillin-clavulanate } \\
\text { Piperacillin-tazobactam }\end{array}$ & $\begin{array}{l}224(65) \\
315(92)\end{array}$ & $\begin{array}{l}64(52) \\
101(83)\end{array}$ & $\begin{array}{l}0.015 \\
0.026\end{array}$ \\
\hline $\begin{array}{l}\text { Carbapenem } \\
\text { Meropenem } \\
\text { Ertapenem }\end{array}$ & $\begin{array}{l}336(98) \\
323(94)\end{array}$ & $\begin{array}{l}119(97) \\
108(88)\end{array}$ & $\begin{array}{l}0.640 \\
0.196\end{array}$ \\
\hline $\begin{array}{l}\text { Other } \\
\text { Ciprofloxacin } \\
\text { Nitrofurantoin } \\
\text { Gentamycin } \\
\text { Trimethoprim/Sulfamethoxazole }\end{array}$ & $\begin{array}{l}93(27) \\
324(94) \\
218(63) \\
146(42)\end{array}$ & $\begin{array}{l}49(40) \\
57(47) \\
95(78) \\
34(28)\end{array}$ & $\begin{array}{l}0.000 \\
0.000 \\
0.000 \\
0.005\end{array}$ \\
\hline
\end{tabular}

Notes: *Statistical significance: $P \leq 0.05$.

Therefore, the difference in serum CRP and Blood NLR between these two ESBL-uropathogens was evaluated in UTI patients (Figure 2). T2DM patients with ESBL-E. coli UTIs had lower serum CRP levels (median, CRP mg/dL 33.7 vs 39.8, respectively; $\mathrm{P}=0.023$ ) and higher blood NLR (median, NLR 3.2 vs 2.6, respectively; $\mathrm{P}=0.010$ ) compared to patients with ESBL-K. pneumoniae UTIs. However, the reverse pattern was observed for nonT2DM patients as they had higher blood NLR levels with ESBL-K. pneumoniae UTIs, but not with ESBL-E. coli UTIs, although not to a significant level (median, NLR 2.8 vs 4.1, respectively; $\mathrm{P}=0.538$ ).

The risk of serum CRP $\geq 35 \mathrm{mg} / \mathrm{dL}$, and blood NLR $\geq 3.5$ was then evaluated for ESBL-E. coli and K. pneumoniae uropathogens in T2DM, and non-T2DM patients. As demonstrated in Figure 3, after adjusting for confounders, including age, gender, serum albumin, hemoglobulin, leukocytes, and platelet counts, T2DM patients with blood NLR $\geq 3.5$ were at higher risk for ESBL-E. coli UTIs [OR, $3.61(95 \% \mathrm{Cl}, 1.49-8.73 ; \mathrm{P}=0.004)]$ than for ESBL- $K$. pneumoniae UTIs. The model showed a good predictive ability for NLR $\geq 3.5(\mathrm{n}=466$, sensitivity $(\%)$ of 88 , specificity (\%) of 73 , positive predictive value (\%) of 93 , and negative predictive value (\%) of 24).

Moreover, although not to a significant level, nonT2DM patients with NLR $\geq 3.5$ were at higher risk for ESBL-K. pneumoniae UTIs, but not for ESBL-E. coli UTIs [OR, $6.11(95 \% \mathrm{Cl}, 0.94-39.67 ; \mathrm{P}=0.058)$ ]

\section{Elevated Urine IL-8 Levels as a Marker of ESBL-E. coli UTIs}

Urinary IL-8 levels were determined for the two groups of T2DM UTI patients using ELISA assay. T2DM patients with ESBL-E. coli UTIs had significantly higher urinary IL-8 levels compared to those with ESBL-K. pneumoniae UTIs (median, IL-8 pg/mL 2120 vs 668.5, respectively; $P<0.0001$ ) (Figure 4).

\section{Survival Analysis}

The risk of all-cause mortality was evaluated for UTI with ESBL-E. coli and $K$. pneumoniae uropathogens and for T2DM, and non-T2DM subgroups. After adjusting for age, gender, and serum albumin, cox proportional hazards regression analysis revealed that T2DM with ESBL-E. coli had higher risk of all-cause mortality than those with ESBL-K. pneumoniae (Hazard ratio [HR], 4.09; 95\%, 1.14-14.59). The risk was non-significant for ESBL-E. coli and $K$. pneumoniae uropathogens in non-T2DM (Figure 5).

\section{Discussion}

This study showed that circulatory inflammatory markers such as serum CRP and blood NLR could differentiate between the type of uropathogen causing UTI in T2DM patients. Elevated blood NLR was shown to correlate with ESBL-E. coli UTIs, but not with ESBL-K. pneumoniae UTIs during T2DM. The reverse trend was observed in non-T2DM, as elevated blood NLR was associated with ESBL-K. pneumoniae UTIs. Subsequently, T2DM patients with ESBL-E. coli UTIs had elevated urinarily IL-8 levels, and higher risk of all-cause mortality compared to those with ESBL-K. pneumoniae UTIs.

ESBL-producing $E$. coli is the causative agent for the majority of UTIs, while only one in four UTIs is caused by 

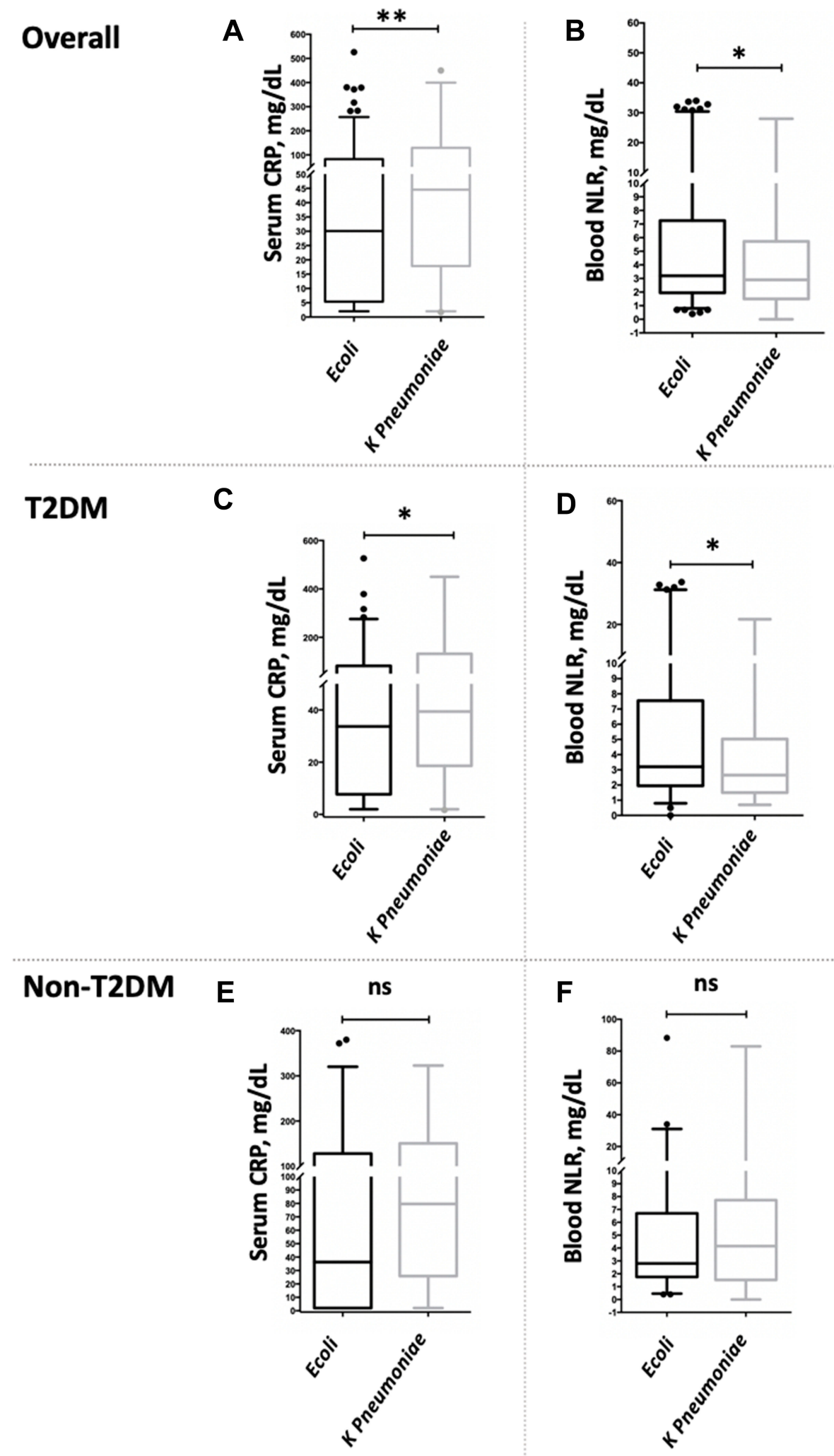

Figure 2 Comparison of circulatory inflammatory markers, including CRPs and NLR between two ESBL-positive UTI groups in accordance with T2DM status. (A, B) Serum CRP and blood NLR levels between E. coli and K. pneumonia in all ESBL-UTI cases. Representative data showing that patients with ESBL-E. coli UTIs had lower serum CRP levels and higher blood NLR compared to ESBL-K. pneumoniae UTIs. (C, D) Serum CRP and blood NLR between E. coli and K. pneumonia in T2DM ESBL-UTI cases. Representative data showing that T2DM patients with ESBL-E. coli UTIs had lower serum CRP levels and higher blood NLR compared to ESBL-K. pneumoniae UTIs. (E, F) Serum CRP and blood NLR between E. coli and K. pneumonia in non-T2DM ESBL-UTI cases. Representative data showing that non-T2DM patients with ESBL-E. coli UTIs had both lower serum CRP levels and blood NLR compared to ESBL-K. pneumoniae UTIs. The values of serum CRP and blood NLR levels were reported in median and IQR. Two-way comparison was done using Mann-Whitney test. $* \mathrm{P}<0.05$, ** $\mathrm{P}<0.00 \mathrm{I}$, ns $=$ non-significant. 


\section{A Serum CRP $\geq 35 \mathrm{mg} / \mathrm{dL}$}

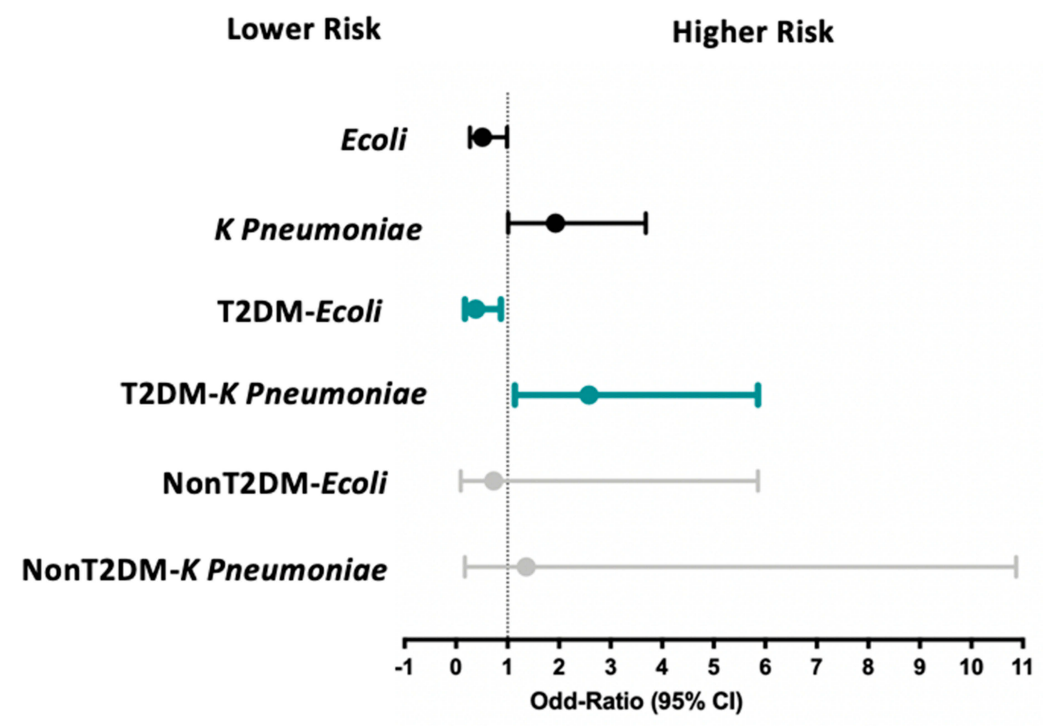

\section{B Blood NLR $\geq 3.5$}

Lower Risk

Higher Risk

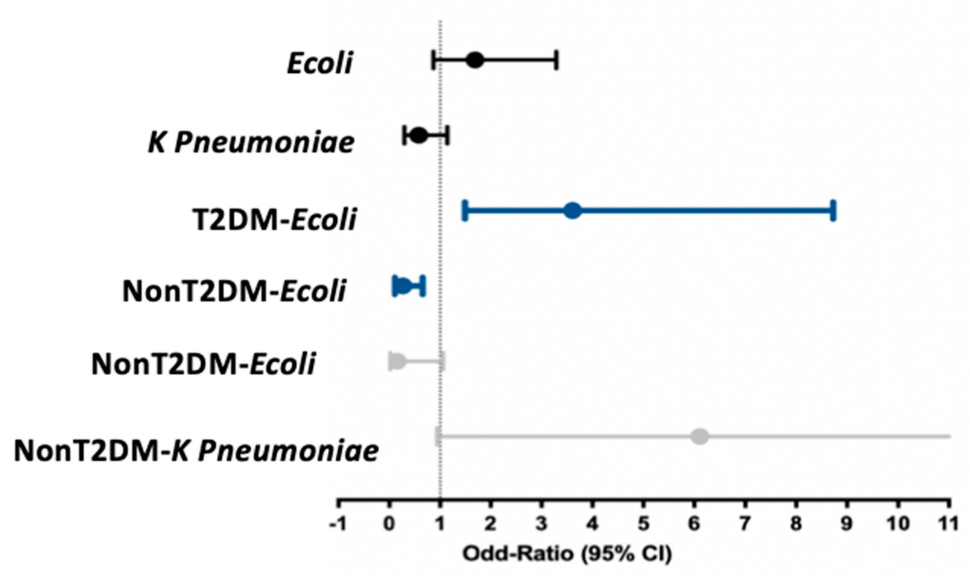

Figure 3 Association of circulatory inflammatory markers, including CRPs and NLR, in the two ESBL-positive UTI groups in accordance with T2DM status. (A) Risk of CRP $\geq$ $35 \mathrm{mg} / \mathrm{dL}$ in all ESBL-UTI cases, T2DM, and non-T2DM. Representative data showing that T2DM patients with serum CRP $\geq 35 \mathrm{mg} / \mathrm{dL}$ were at higher risk for ESBL-E. coli UTIs than for ESBL-K. pneumoniae UTIs. (B) Risk of NLP $\geq 35 \mathrm{mg} / \mathrm{dL}$ in all ESBL-UTI cases, T2DM, and non-T2DM. Representative data showing that T2DM patients with blood NLR $\geq 3.5$ were at higher risk for ESBL-E. coli UTIs than for ESBL-K. pneumoniae UTIs. Adjusted for age, gender, serum albumin, hemoglobulin, leukocytes, and platelet counts.

ESBL-producing K. pneumoniae. ${ }^{1}$ It is, hence, crucial that we understand its role in the subversion of host innate immunity. We found that uropathogenic E. coli was associated with lower serum inflammatory markers measured as serum CRP level compared to $K$. pneumoniae. This difference in the level of CRP between the two uropathogens is indicative of the difference in innate-immune responses initiated against these uropathogens. Recently, it has been reported that uropathogenic $E$. coli subverts the host innate- immune system by causing zinc mediated toxicity to persist and disseminate within the host. ${ }^{16}$ An inverse relationship between serum CRP levels and zinc was acutely reported in UTI patients. ${ }^{17}$ Therefore, our finding that CRP levels are downregulated during $E$. coli UTI compared to $K$. pneumoniae is in line with the reported pathogenesis mechanisms of E. coli. Clinically, K. pneumoniae, which cause the highest acute inflammatory response measured as elevated CRP may rarely cause renal scars. ${ }^{18,19}$ 


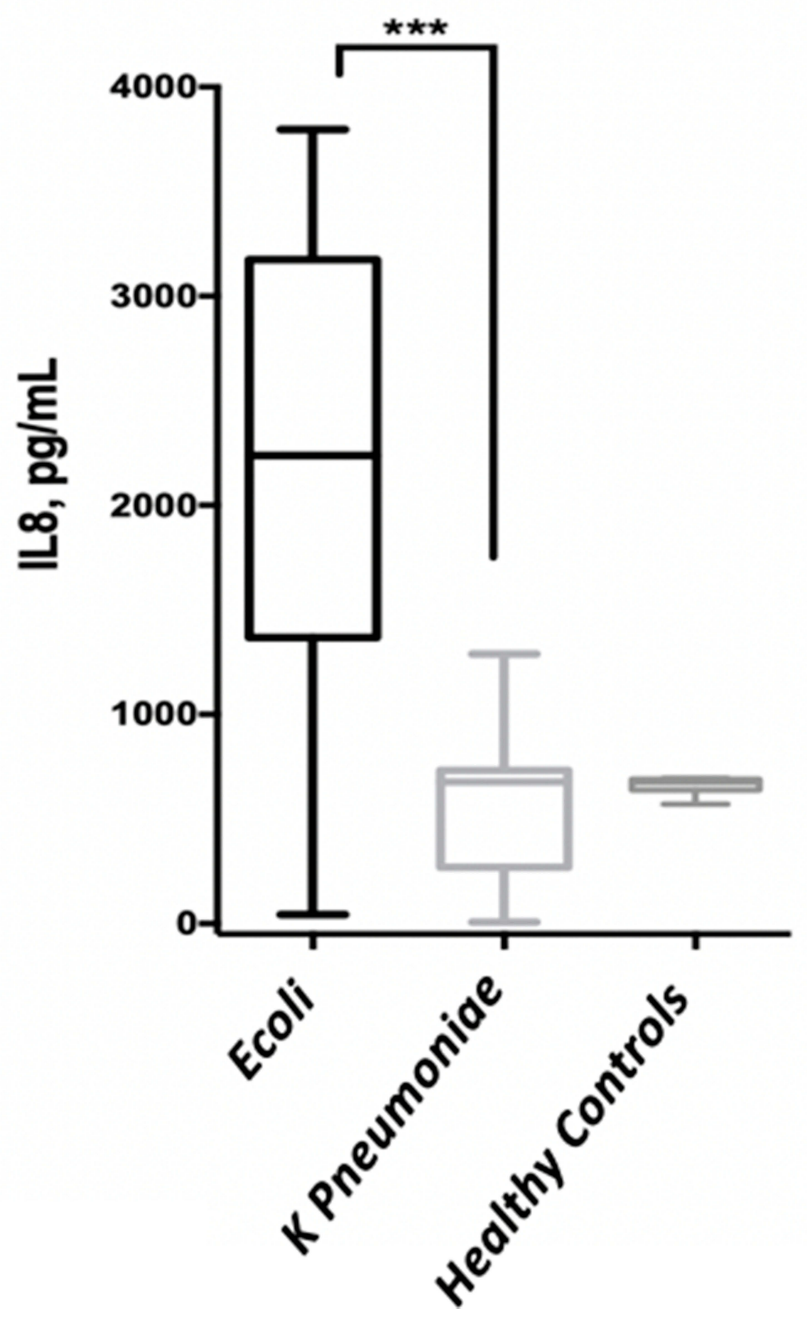

Figure 4 Comparison of urinary IL-8 level between the two ESBL-positive UTI groups in T2DM. **** $\mathrm{P}<0.0001$.

T2DM patients with ESBL-E. coli UTIs were associated with higher serum NLR and elevated urinary IL-8 level compared to those with ESBL- positive $K$. pneumoniae UTIs. During infection with uropathogenic E. coli, mature neutrophils circulate through the blood and enter infected uroepithelium where they impose their neutrophilmediated bactericidal activity, or phagocytosis, ${ }^{20,21}$ and induce the release of IL-8 for surrounding tissue cells. IL-8 was also shown to be released by mature cells themselves upon phagocyting bacteria. ${ }^{22}$

Furthermore, gene-set enrichment analysis (GSEA) of human neutrophils incubated with $E$. coli indicated that this uropathogen induced an upregulation of IL-8 signaling within neutrophils (Figure S1). This suggested that neutrophils could be an important source of its own chemoattractant. In T2DM, the increase in blood NLR and urinary IL-8 is of concern as high IL- 8 can increase the progression of renal disease. ${ }^{23,24}$
Renal damage and scarring are reported to be due to cytotoxic products of neutrophils. ${ }^{25}$ A positive correlation between NLR and diabetic peripheral neuropathy has been recently suggested. ${ }^{26}$ Therefore, correlating NLR to urinary IL-8 levels could suggest that these markers could be used as an independent risk factor for diabetic nephropathy. Furthermore, IL-8induced by uropathogenic E. coli could exacerbate urinary tract tissue inflammation of T2DM patients; resulting in tissue damage and lower overall survival.

On the contrary blood, NLR were not elevated in nonT2DM during E. coli UTI (uropathogenic E. coli caused a reduction in blood NLR in non-T2M). Clinically, there is a need for a diagnostic biomarker for UTI, especially in T2DM. Other diagnostic biomarkers of bacterial UTIs such as serum procalcitonin ${ }^{27}$ and $\mathrm{CRP}^{28}$ are either non-specific for uropathogens, or cannot differentiate between them. The reverse pattern of blood NLR in non-T2DM patients supports the predictive ability of this blood marker for $E$. coli UTI in T2DM.

Uropathogenic $E$. coli and $K$. pneumoniae can develop antibiotic resistance that may lead to end-organ damage, especially in immunocompromised patients such as in T2DM. ${ }^{29}$ The fact that uropathogenic E. coli and K. pneumoniae subvert the innate-immune system via different mechanisms suggest that the pathological damage they induce could also be different. ESBL-E. coli UTIs was associated with higher all-cause mortality specifically in T2DM patients. The observed blood NLR as well as IL-8 levels could contribute, besides bacterial persistence, to enhanced nephrotic inflammations, and the observed higher mortality in T2DM patients. This suggest that using immune suppressive agents, besides antibiotics, may improve the clinical outcome of these diabetics, and reduce their mortality rate.

An intrinsic limitation of this study is not a randomized clinical trial. By using a multivariate logistic regression model, we were able to adjust for the observed confounders and assess the inflammatory signatures indicative of the two uropathogens. However, there might be a number of unobservable factors that could only be controlled with a randomized controlled trial. Another limitation of this study is the small sample size, which is due to the single-institutional nature of the study. Clinical validation needs to be carried out on a larger cohort. Finally, in this study, we were unable to investigate the molecular mechanisms regulating the observed difference in inflammatory profiles between the two groups, or their molecular contribution to the overall pathogenesis and clinical outcome of the two UTI pathogenic groups. This is mostly because we did not have access to blood samples from these patients. Future studies addressing 


\section{A Overall}

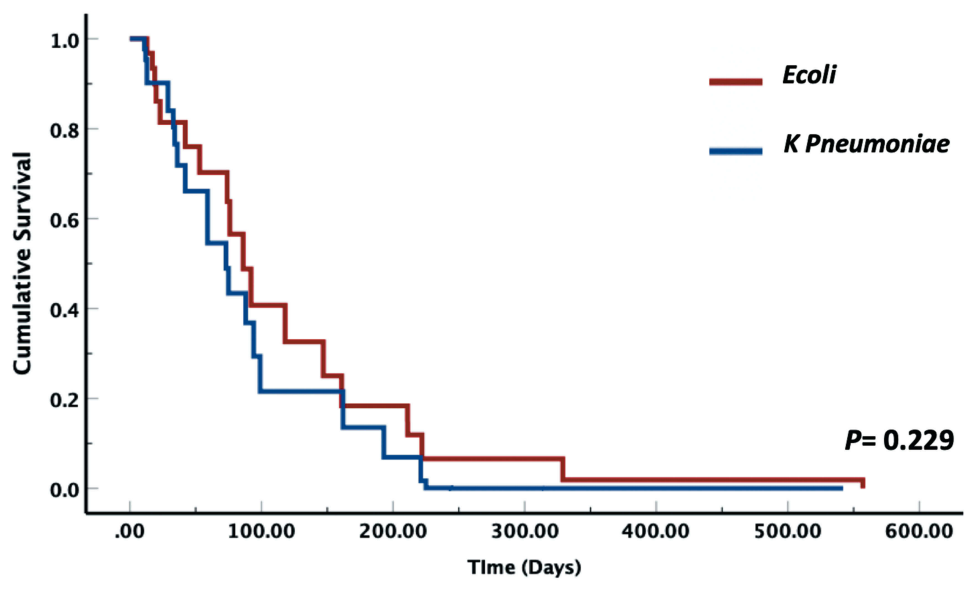

\section{B T2DM}

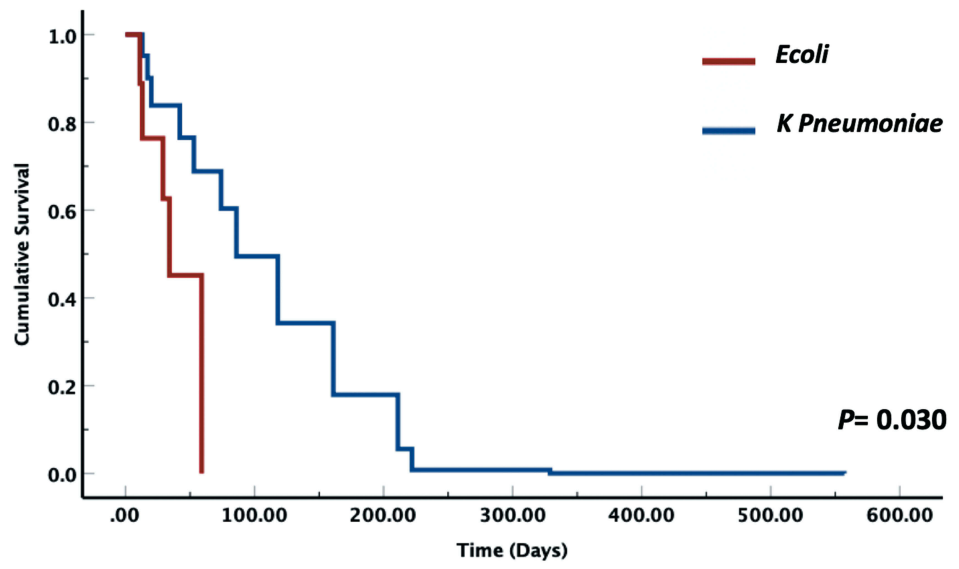

\section{C non-T2DM}

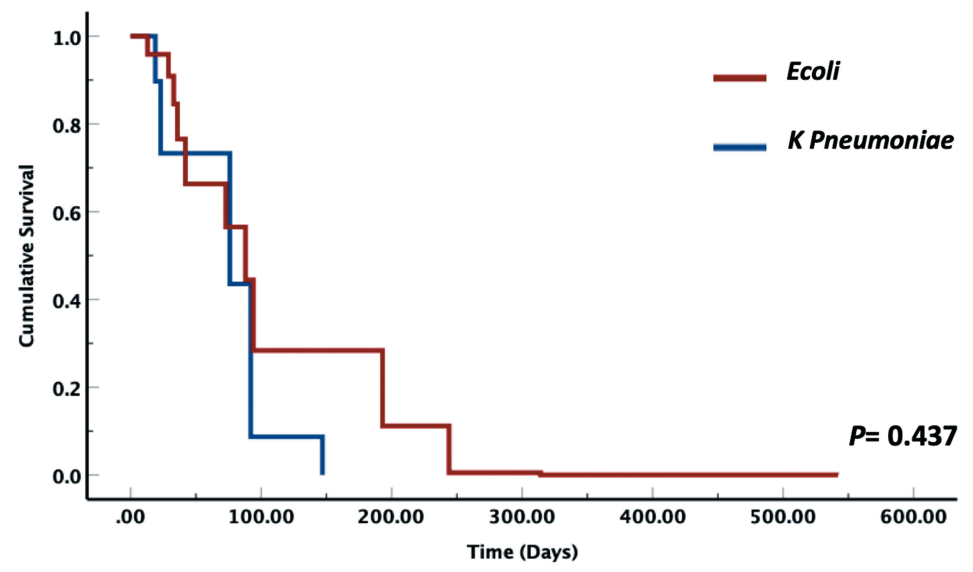

Figure 5 Hazard ratios and cumulative survival curve in the two ESBL-positive UTI groups in accordance with T2DM status. (A) Risk estimate for all-cause mortality between $E$. coli and $K$. pneumonia in all ESBL-UTI cases. Representative data showing that the risk was non-significant for ESBL-E. coli and K. pneumoniae uropathogens in all ESBL-UTI cases. (B) Risk estimate for all-cause mortality between $E$. coli and $K$. pneumonia in T2DM ESBL-UTI cases. Representative data showing that T2DM with ESBL-E. coli had higher risk of all-cause mortality than those with ESBL-K. pneumoniae. (C) Risk estimate for all-cause mortality between E. coli and $K$. pneumonia in non-T2DM ESBLUTI cases. Representative data showing that the risk was non-significant for ESBL-E. coli and K. pneumoniae uropathogens in non-T2DM. 
these mechanisms may highlight important therapeutic targets that could lead to better control of UTIs, especially in immunocompromised T2DM patients.

In conclusion, we have shown that in T2DM patients, a combination of serum CRP, blood NLR, and urinary IL-8 levels could serve as a biomarker for ESBL-E. coli UTI. Moreover, combining antibiotic treatment with immunosuppressive drugs might improve the clinical outcomes as well as the survival of T2DM patients with ESBL-E. coli UTIs.

\section{Funding}

This research has been financially supported by Sharjah Research Academy (Grant code: MED001); University of Sharjah (Grant code: 1901090254); Tissue Injury and Repair (TIR) group operational grant; by a seed grant to Rabih Halwani, University of Sharjah; and by Prince Abdullah Ben Khalid Celiac Disease Research Chair, under the Vice Deanship of Research Chairs, King Saud University, Riyadh, Kingdom of Saudi Arabia.

\section{Disclosure}

Rabih Halwani and Rifat Hamoudi are co-senior authors. All authors declare that they have no competing interests.

\section{References}

1. Petty NK, Ben Zakour NL, Stanton-Cook M, et al. Global dissemination of a multidrug resistant Escherichia coli clone. Proc Natl Acad Sci. 2014;111(15):5694-5699. doi:10.1073/pnas.1322678111

2. Pitout JD, Nordmann P, Laupland KB, Poirel L. Emergence of Enterobacteriaceae producing extended-spectrum $\beta$-lactamases (ESBLs) in the community. $J$ Antimicrob Chemother. 2005;56(1):5259. doi:10.1093/jac/dki166

3. Rodriguez-Baño J, Paterson DL. A change in the epidemiology of infections due to extended-spectrum $\beta$-lactamase-producing organisms. Clin Infect Dis. 2006;42(7):935-937. doi:10.1086/500945

4. Harris PN, Tambyah PA, Paterson DL. $\beta$-lactam and $\beta$-lactamase inhibitor combinations in the treatment of extended-spectrum $\beta$-lactamase producing Enterobacteriaceae: time for a reappraisal in the era of few antibiotic options? Lancet Infect Dis. 2015;15(4):475-485. doi:10.1016/S1473-3099(14)70950-8

5. Hamdan Z, Kubbara E, Adam AM, Hassan OS, Suliman SO, Adam I. Urinary tract infections and antimicrobial sensitivity among diabetic patients at Khartoum, Sudan. Ann Clin Microbiol Antimicrob. 2015;14 (1):26. doi:10.1186/s12941-015-0082-4

6. Rahim MA, Mitra P, Haque A, et al. Urinary tract infection due to extended-spectrum beta-lactamase producing organisms is a risk factor for acute kidney injury among patients with type 2 diabetes mellitus. $J$ Med. 2017;19(1):40-43. doi:10.3329/jom.v19i1.34840

7. Liu A, Bui T, Van Nguyen H, Ong B, Shen Q, Kamalasena D. Serum C-reactive protein as a biomarker for early detection of bacterial infection in the older patient. Age Ageing. 2010;39(5):559-565. doi:10.1093/ageing/afq067

8. Shaikh N, Borrell JL, Evron J, Leeflang MM. Procalcitonin, C-reactive protein, and erythrocyte sedimentation rate for the diagnosis of acute pyelonephritis in children. Cochrane Database Syst Rev. 2015;1: CD009185.
9. Han SY, Lee IR, Park SJ, Kim JH, Shin JI. Usefulness of neutrophillymphocyte ratio in young children with febrile urinary tract infection. Korean J Pediatr. 2016;59(3):139-144. doi:10.3345/kjp.2016. 59.3.139

10. Gökhan Ş, Tanrıverdi F, Günaydın GP, et al. Importance of thiol/ disulfide homeostasis and the neutrophil-to-lymphocyte ratio in diagnosing of urinary tract infections in the emergency department. Cyprus J Med Sci. 2019;4(1):19-23. doi:10.5152/cjms.2019.704

11. Tramma D, Hatzistylianou M, Gerasimou G, Lafazanis V. Interleukin-6 and interleukin-8 levels in the urine of children with renal scarring. Pediatr Nephrol. 2012;27(9):1525-1530. doi:10.1007/ s00467-012-2156-2

12. Alberti KG, Zimmet PZ. Definition, diagnosis and classification of diabetes mellitus and its complications. Part 1: diagnosis and classification of diabetes mellitus. Provisional report of a WHO consultation. Diabet Med. 1998;15(7):539-553. doi:10.1002/(SICI)1096-9136 (199807) 15:7<539::AID-DIA668>3.0.CO;2-S

13. Faul F, Erdfelder E, Buchner A, Lang AG. Statistical power analyses using $\mathrm{G}^{*}$ power 3.1: tests for correlation and regression analyses. Behav Res Methods. 2009;41(4):1149-1160. doi:10.3758/BRM.41.4. 1149

14. Denholtz M, Murre C RNA-seq profiling of the human neutrophil genome during PMA- and E. coli encounter-induced activation. Gene expression omnibus. Available from: https:/www.ncbi.nlm.nih.gov/ geo/query/acc.cgi?acc=GSE126757. Accessed May 29, 2020.

15. Hamoudi RA, Appert A, Ye H, et al. Differential expression of NF$\kappa \mathrm{B}$ target genes in MALT lymphoma with and without chromosome translocation: insights into molecular mechanism. Leukemia. 2010;24 (8):1487-1497. doi:10.1038/leu.2010.118

16. Stocks CJ, Phan M-D, Achard MES, et al. Uropathogenic Escherichia coli employs both evasion and resistance to subvert innate immunemediated zinc toxicity for dissemination. Proc Natl Acad Sci. 2019;116(13):6341-6350. doi:10.1073/pnas.1820870116

17. Craig GM, Evans SJ, Brayshaw BJ. An inverse relationship between serum zinc and C-reactive protein levels in acutely ill elderly hospital patients. Postgrad Med J. 1990;66(782):1025-1028. doi:10.1136/ pgmj.66.782.1025

18. de Man P, Cläeson I, Johanson I, Jodal U, Svanborg Edén C. Bacterial attachment as a predictor of renal abnormalities in boys with urinary tract infection. $J$ Pediatr. 1989;115(6):915-922. doi:10.1016/S0022-3476(89)80742-5

19. Mundi H, Björkstén B, Svanborg C, Ohman L, Dahlgren C. Extracellular release of reactive oxygen species from human neutrophils upon interaction with Escherichia coli strains causing renal scarring. Infect Immun. 1991;59(11):4168-4172.

20. Borregaard N. Neutrophils, from marrow to microbes. Immunity. 2010;33(5):657-670. doi:10.1016/j.immuni.2010.11.011

21. Kolaczkowska E, Kubes P. Neutrophil recruitment and function in health and inflammation. Nat Rev Immunol. 2013;13:159-175.

22. Bazzoni F, Cassatella MA, Rossi F, Ceska M, Dewald B, Baggiolini M. Phagocytosing neutrophils produce and release high amounts of the neutrophil-activating peptide 1/interleukin 8. J Exp Med. 1991;173(3):771-774. doi:10.1084/jem.173.3.771

23. Zhao D, Kuhnt-Moore S, Zeng H, Wu JS, Moyer MP, Pothoulakis C. Neurotensin stimulates IL-8 expression in human colonic epithelial cells through Rho GTPase-mediated NF-kB pathways. Am J Physiol Cell Physiol. 2003;284(6):C1397-404. doi:10.1152/ajpcell.00328. 2002

24. Khandare SA, Chittawar S, Nahar N, Dubey TN, Qureshi Z. Study of neutrophil-lymphocyte ratio as novel marker for diabetic nephropathy in type 2 diabetes. Indian J Endocrinol Metab. 2017;21(3):387-392. doi:10.4103/ijem.IJEM_476_16

25. Roilides E, Papachristou F, Gioulekas E, et al. Increased urine interleukin- 6 concentrations correlate with pyelonephritic changes on $99 \mathrm{~m}$ Tc-dimercaptosuccinic acid scans in neonates with urinary tract infections. J Infect Dis. 1999;180(3):904-907. doi:10.1086/314960 
26. Xu T, Weng Z, Pei C, et al. The relationship between neutrophil-tolymphocyte ratio and diabetic peripheral neuropathy in type 2 diabetes mellitus. Medicine. 2017;96(45):e8289. doi:10.1097/MD.0000 000000008289

27. Levine AR, Tran M, Shepherd J, Naut E. Utility of initial procalcitonin values to predict urinary tract infection. Am J Emerg Med. 2018;36(11):1993-1997. doi:10.1016/j.ajem.2018.03.001
28. Kuil SD, Hidad S, Fischer JC, et al. Sensitivity of point-of-care testing $\mathrm{C}$ reactive protein and procalcitonin to diagnose urinary tract infections in Dutch nursing homes: PROGRESS study protocol. BMJ Open. 2019;9(8):e031269. doi:10.1136/bmjopen-2019-031269

29. Casqueiro J, Casqueiro J, Alves C. Infections in patients with diabetes mellitus: a review of pathogenesis. Indian J Endocrinol Metab. 2012;16(Suppl1):S27-36. doi:10.4103/2230-8210.94253

\section{Publish your work in this journal}

Infection and Drug Resistance is an international, peer-reviewed openaccess journal that focuses on the optimal treatment of infection (bacterial, fungal and viral) and the development and institution of preventive strategies to minimize the development and spread of resistance. The journal is specifically concerned with the epidemiology of antibiotic resistance and the mechanisms of resistance development and diffusion in both hospitals and the community. The manuscript management system is completely online and includes a very quick and fair peerreview system, which is all easy to use. Visit http://www.dovepress.com/ testimonials.php to read real quotes from published authors. 